\title{
Frontières
}

\section{Points de convergences et perspectives interdisciplinaires sur le soulagement de la douleur et la souffrance}

\section{Jocelyne Saint-Arnaud}

Volume 17, numéro 2, printemps 2005

Surtout, ne pas souffrir

URI : https://id.erudit.org/iderudit/1073482ar

DOI : https://doi.org/10.7202/1073482ar

Aller au sommaire du numéro

Éditeur(s)

Université du Québec à Montréal

ISSN

1180-3479 (imprimé)

1916-0976 (numérique)

Découvrir la revue

Citer ce document

Saint-Arnaud, J. (2005). Points de convergences et perspectives

interdisciplinaires sur le soulagement de la douleur et la souffrance. Frontières, 17(2), 5-6. https://doi.org/10.7202/1073482ar d'utilisation que vous pouvez consulter en ligne. 


\section{Points de convergences et perspectives interdisciplinaires sur le soulagement de la douleur et la souffrance}

Jocelyne Saint-Arnaud, Ph.D., responsable du thème.

La société occidentale occulte aussi bien l'expérience de la mort, que la douleur et la faiblesse qui l'accompagnent. Combien de personnes ont été choquées de voir le pape Jean-Paul II montrer ses problèmes de santé en direct? N'y avait-il pas là un message, que chacun peut interpréter à sa façon, indiquant que la douleur et la souffrance existent et qu'elles sont porteuses de sens. À l'opposé, les mythes contemporains véhiculés par les médias, par la publicité, mais aussi par des institutions et des découvertes scientifiques, valorisent tout ce qui promeut la santé corporelle et la beauté physique, tout ce qui permet de prolonger la vie, qu'il s'agisse de techniques et procédés scientifiquement éprouvés ou de moyens fantaisistes et farfelus. Face à l'idéal mythique de la personne jeune, belle et en santé, la douleur et, pire, la souffrance, apparaissent hors circuit, échecs des sociétés riches en quête d'immortalité.

Ce numéro de la revue Frontières portant sur le soulagement de la douleur et la souffrance regroupe des auteurs provenant tant des sciences humaines que des sciences de la santé pour explorer un sujet qui se présente comme une problématique commune à tous les temps. L'anthropologie (Le Breton), la médecine (Beauchamp, Vinay et ses collègues), le droit (Mullins), les sciences infirmières (Alderson, Hudon, Lefebvre et Levert, Savoie et Le May), la pharmacie (Lachaine, Néron), la philosophie (Dumais, Plourde, Saint-Arnaud), la théologie (Jacquemin), l'exégèse (Lavoie), la peinture et la poésie (Duchesne) y apportent leurs perspectives et connaissances. L'ensemble constitue un apport unique à la compréhension des phénomènes à l'étude.

Parler de douleur et de souffrance, c'est d'une certaine manière endosser le dualisme cartésien, toujours prégnant dans la culture occidentale, la douleur étant associée à une atteinte physique, la souffrance à une atteinte psychique, voire morale. Or, dira David Le Breton, «la douleur n'écrase pas le corps, elle écrase l'individu, elle rompt l'évidence de son rapport au monde, elle brise l'écoulement de la vie quotidienne et altère la relation aux autres». C'est donc tout l'être que la douleur monopolise. Reprenant Saunders, certains (Beauchamp, Saint-Arnaud) parleront à ce sujet de douleur totale, celle qui menace l'intégrité de l'être, le sens de l'humain en chacun. D'autres nomment souffrance globale la tendance qu'ont les composantes physiques, psychologiques, affectives, spirituelles et sociales, d'interagir et de s'intensifier les unes les autres (Plourde). Les concepts de douleur et de souffrance se rejoignent. Parfois, ils se présentent comme les deux faces d'un même phénomène. Mais, plus profonde et d'un spectre plus large, la souffrance est liée à la signification que l'individu donne à sa douleur. «Ce ne sont pas tant les circonstances qui induisent de la souffrance que la manière dont elles sont interprétées et vécues » (Le Breton), d'où la subjectivité de la souffrance qui prendra de multiples visages selon les individus, les groupes et les cultures. Cependant, des personnes et des groupes vivent l'invivable, l'indicible, des situations qu'aucune culture ne saurait accepter parce que dégradantes pour tout être humain. C'est le cas des femmes afghanes dont nous entretient, entre autres injustices, Monique Dumais.

Si la douleur peut s'objectiver et être évaluée, la souffrance s'y refuse. Ce qu'on en sait repose sur les indications qu'en donne l'individu souffrant. La parole souffrante s'exprime dans un témoignage dira Jean-Jacques Lavoie à partir de l'analyse de textes anciens provenant des trois religions monothéistes. Et il s'agit là d'un premier thème sur lequel il y a convergence de la part des auteurs de ce numéro: l'importance que la souffrance soit dite. La parole libère, met le phénomène à distance et crée des liens. Selon Levinas, la souffrance se réduit en dernier ressort à la solitude de l'individu (Plourde). Cette solitude peut être perçue dans les tableaux de Raymonde Duchesne. Les personnes y sont isolées, figées dans un triste avenir. Plourde nous rappelle que, pour Levinas, la solitude fait référence au lien que chacun entretient avec lui-même. À la limite, celui qui souffre devient étranger à lui-même. 
Le «patient» n'est pas seul à souffrir. Plusieurs auteurs traitent de la souffrance des soignants. Dominique Jacquemin explique ce phénomène par l'écart entre un idéal de soin et la réalité de la prise en charge. Selon Marie Alderson, la principale source de souffrance pour les infirmières provient «de la perception de douloureux manques de reconnaissance, d'autonomie et de pouvoir professionnels». Hélène Lefebvre et Marie-Josée Levert relient la souffrance des soignants à leur impuissance à répondre de manière adéquate à la souffrance d'autrui. Elles montrent comment il est difficile pour les professionnels de la santé d'être porteurs de mauvaises nouvelles, de faire l'annonce d'un diagnostic sombre, de dire l'indicible, compte tenu de la difficulté à prévoir l'évolution d'un traumatisme craniocérébral et à modifier le cours de la maladie. Face aux professionnels, les familles souffrent; elles sont paralysées par cette annonce; elles vivent elles-mêmes un véritable choc traumatique "qui se manifeste par un état de stupeur et de sidération dont l'intensité varie [...] mais qui laissera une cicatrice indélébile».

Mais le «patient», entendons celui qui souffre, n'est pas nécessairement passif; il peut aussi être résistant, selon l'expression de David Le Breton, ce que l'on retrouve aussi dans l'interprétation exégétique de l'histoire de Job par JeanJacques Lavoie et dans l'analyse d'Yvon Beauchamp. Ce dernier décrit la douleur comme un mécanisme de défense ayant pour fonction le maintien de l'intégrité physique de la personne face à son environnement et le maintien d'une intégrité interne. On retrouve donc au plan physiologique, ce que l'anthropologue et l'exégète confirment dans leurs perspectives disciplinaires. Tout le travail du soignant ne serait-il pas dans la transformation du patient passif en patient résistant, que ce soit aux plans physique ou psychologique et social pour permettre, notamment par le moyen de l'empowerment, une réintégration de la personne dans la communauté des humains.

À ce sujet, le mythe d'Esculape présenté par Patrick Vinay et ses collègues est instructif à plus d'un égard. Le dieu de la médecine, lui-même blessé, amène le malade à renoncer à son état antérieur pour "accéder à un nouvel ordre des choses où sa blessure [sera] désormais intégrée à sa réalité». Le médecin et le patient cheminent ensemble vers un nouvel équilibre. Ce phénomène est étudié par Hélène Lefebvre et sa collègue sous le nom de résilience. S'inspirant des résultats de leurs recherches, les auteures proposent un modèle d'intervention qui tient compte des forces de chacun. Il s'agit d'un partenariat à établir entre les différents acteurs qui intègre le deuil d'un état de santé antérieur et permet de découvrir de nouvelles forces jusque-là insoupçonnées. En fait, chacun s'engage dans un processus de transformation personnelle pour construire un nouvel équilibre, malgré et au-delà de la perte. Partager l'expérience de souffrance d'autrui met la créativité à l'épreuve, ce que confirme Dominique Jacquemin à propos de solutions éthiques apportées par les équipes de soins aux problèmes et dilemmes rencontrés.

Pour un grand nombre d'auteurs, sinon tous, la douleur ne saurait être que physique. Comme l'écrit Yvon Beauchamp, "les phénomènes douloureux, surtout chroniques, font toujours appel à la globalité des systèmes de l'humain et ne vouloir traiter que des symptômes physiques est le meilleur chemin vers l'insuccès ». Au-delà des interventions techniques, le soulagement de la douleur devrait s'adjoindre dans tous les cas, une démarche d' accompagnement, qui brise la solitude et l'incompréhension.

Mais, et c'est là que se heurtent deux cultures, celle d'une approche technique et scientifique (au sens moderne du terme) qui remonte au développement de la médecine expérimentale (notamment avec Roger Bacon), décrite comme très interventionniste et ayant pour but de vaincre la nature, et celle d'une approche globale centrée sur le patient qui date des temps les plus reculés, comme le montrent Vinay et ses collègues. Dit autrement, c'est le choc de deux modèles que Dominique Jacquemin considère inconciliables: un modèle médico-technique et un modèle holistique, souvent identifiés, pour le premier, à l'intervention médicale et, pour le deuxième, à l'intervention infirmière. Il est en effet difficile pour les soignants de concilier ces deux modèles et ceci se vérifie particulièrement bien dans le domaine du soulagement de la douleur.

En fait, des barrières se dressent tant dans le développement de chacune de ces deux cultures que dans la reconnaissance de leur complémentarité quant au soulagement adéquat de la douleur. De fausses croyances subsistent chez les intervenants, les bénéficiaires et leurs familles, concernant l'utilisation des narcotiques. Des mythes persistants sont ébranlés à la lecture de ce numéro: la douleur n'est qu'un phénomène physique (Le Breton, Beauchamp, Plourde); les produits naturels n'interagissent pas avec les médicaments (Néron), le soulagement de la douleur engendre une mort plus rapide (Saint-Arnaud), le soulagement de la douleur donnerait lieu à des poursuites devant les tribunaux (Mullins), le sous-traitement de la douleur n'entraîne pas de coûts financiers importants (Lachaine), les soignants ne souffrent pas (Alderson, Jacquemin, Lefebvre et Levert). Par ailleurs, plusieurs auteurs de ce numéro (Beauchamp, Hudon, Jacquemin, Saint-Arnaud, Savoie et Le May) reconnaissent des lacunes dans la formation disciplinaire à l'égard du soulagement de la douleur. Ainsi, les infirmières manquent de connaissances notamment en neurophysiologie de la douleur et en pharmacologie, comme le montrent les résultats de l'étude de Maryse Savoie et Sylvie Le May. Par contre, la nécessité de mettre en place des programmes intégrés de $1^{\text {er }}$ et $2^{\mathrm{e}}$ cycle, permettant une formation théorique et clinique en soulagement de la douleur (Beauchamp), met en évidence les carences dans la formation médicale actuelle, notamment pour ce qui concerne la communication avec les patients et leur famille. Les institutions universitaires et hospitalières ont une responsabilité morale en cette matière. Enfin, l'interdisciplinarité est souvent problématique (Alderson), voire inexistante, d'où l'inquiétude d'Andrée Néron qui montre comment les interactions médicamenteuses peuvent avoir de graves conséquences sur le traitement de la douleur, voire engendrer de la douleur, ce qui est trop souvent ignoré par les médecins, avec les risques que cela comporte.

Malgré tout, l'exemple des soins palliatifs nous conforte dans la possibilité d'intégrer une approche scientifique à une approche globale du soin. La bonne mort fait plus appel à de bons soins qu'à la possibilité d'un recours à l'euthanasie ou à une aide au suicide, actes qui relèvent de la technique. L'approche globale s'effectue à partir d'une intervention multidisciplinaire qui n'est pas la somme d'interventions techniques effectuées par les différentes disciplines impliquées, mais qui repose sur des qualités éthiques d'ouverture à l'autre dans un partenariat à construire entre les membres des équipes de soins, d'une part, et entre les équipes de soins et les bénéficiaires et leurs familles, d'autre part. À cet égard, le partage d'une philosophie commune centrée sur le bien-être du malade, ainsi que l'inclusion de la dimension éthique dans les pratiques constituent des éléments intégrateurs. 